\title{
Tarifpolitischer Jahresbericht 2006: Aufwärtstrend bei Abschlüssen - Konflikte um Beschäftigungssicherung und Tarifstandards
}

Die Tarifabschlüsse des Jahres 2006 sind in vielen Branchen erkennbar besser ausgefallen als im Vorjahr. Insbesondere die Abkommen in der Metall- und Stahlindustrie signalisierten eine Trendwende. Doch noch wirken die lang laufenden, sehr niedrigen Abschlüsse aus dem Vorjahr dämpfend auf die gesamtwirtschaftliche Lohnbilanz. Das ist das Fazit der Lohnrunde 2006. Neben den Lohnverhandlungen bestimmten der Tarifkonflikt im öffentlichen Dienst und im Gesundheitswesen sowie Konflikte um Beschäftigungs- und Standortsicherung, z. B. bei Volkswagen oder bei der AEG in Nürnberg, das Tarifgeschehen. Die politische Diskussion um die angemessene tarifliche und/oder gesetzliche Sicherung von Mindestlöhnen führte im vergangenen Jahr zu keinem konkreten Ergebnis.

\section{Das Tarifjahr 2006 im Überblick}

\section{1 ÖKONOMISCHE UND POLITISCHE RAHMENBEDINGUNGEN}

Die ökonomischen Rahmenbedingungen entwickelten sich im vergangenen Jahr deutlich besser als von nahezu allen Experten und Wirtschaftsinstituten erwartet. Der Sachverständigenrat schätzte im Herbst 2005 das reale Wachstum für 2006 auf lediglich 1,0\%. Die Institute lagen mit einer Wachstumsprognose von 1,2\% nur unwesentlich höher. Für den Arbeitsmarkt wurde allenfalls eine leichte Belebung erwartet, die Zahl der registrierten Arbeitslosen sollte gerade einmal um knapp 100.000 zurückgehen. Im Laufe der Monate korrigierten alle Beteiligten ihre Prognosen schrittweise nach oben und blieben dennoch hinter der tatsächlichen Entwicklung zurück. Das Bruttoinlandsprodukt wuchs im Jahr 2006 real um 2,5 \%. Die wesentlichen Wachstumsimpulse kamen wiederum vom Export, der in zweistelliger Größenordnung zunahm, aber auch unerwartet stark von den (privaten) Bruttoinvestitionen, die um mehr als $6 \%$ stiegen. Mehr als bescheiden blieb erwartungsgemäß der private Konsum, der mit einem realen Plus von $0,6 \%$ knapp über der Nulllinie lag. Auch am Arbeitsmarkt deutete sich eine kleine Wende an: Die Zahl der sozialversicherungspflichtigen Beschäftigten stieg erstmals nach Jahren wieder an. Im Jahres- durchschnitt ging die Zahl der Arbeitslosen um rund 0,35 Mio. auf 4,5 Mio. zurück, die Arbeitslosenquote sank um 0,9 auf 10,8\%. Zum Teil hängt dies allerdings mit Veränderungen in der statistischen Erfassung zusammen. Die Gewinnsituation der Unternehmen verbesserte sich erneut: Die Unternehmens- und Vermögenseinkommen stiegen nach knapp $12 \%$ und 6,2 \% in den Jahren 2004 und 2005 im vergangenen Jahr noch einmal um 6,9\%. Die Tarifrunde startete also in einer Phase konjunktureller Belebung, die allerdings die exportorientierten Sektoren sehr viel stärker erfasste als die binnenmarktorientierten Bereiche.

Nach der Bundestagswahl im Herbst 2005 und dem Antritt der Großen Koalition aus CDU/CSU und SPD unter Kanzlerin Angela Merkel war die politische Großwetterlage deutlich verändert. Unmittelbare Angriffe auf die Gewerkschaften, die bereits in der Vorwahlkampfzeit deutlich zurückgegangen waren, unterblieben völlig. Das politische Klima im Umfeld der Tarifrunde war für die Gewerkschaften sogar durchaus günstig. Politiker von SPD und Union sprachen sich für angemessene Lohnsteigerungen aus. Hintergrund waren die dahindümpelnde Binnenwirtschaft, die ausgesprochen positive Gewinnentwicklung in zahlreichen DAX-Konzernen und das in der Öffentlichkeit verbreitete Gefühl, dass nach Jahren der Lohnzurückhaltung nun auch die ArbeitnehmerInnen einen Anteil an der wirtschaftlichen Entwicklung erhalten sollten.

Im Mittelpunkt der politischen Diskussion stand die Ausgestaltung der Arbeitsmarkt- und Sozialpolitik; insbesondere die
Gestaltung des Niedriglohnsektors (Kombilohn) und die Sicherung von Mindeststandards bei den Erwerbseinkommen (gesetzlicher Mindestlohn) waren (und sind) zwischen und innerhalb der Koalitionsparteien und den Gewerkschaften strittig.

\subsection{FORDERUNGEN UND ABSCHLÜSSE}

Aufgrund von mehrjährigen Abschlüssen im Jahr 2005 nahmen nicht alle Branchen an der Lohnrunde 2006 teil; so laufen zum Beispiel die Tarifabkommen aus dem Jahr 2005 in den Bereichen Druckindustrie, Bauhauptgewerbe, Versicherungsgewerbe, Groß- und Außenhandel zum Teil bis ins Jahr 2007. Im öffentlichen Dienst (Bund, Gemeinden) reicht die Laufzeit sogar bis Ende 2007.

Die IG Metall forderte für die Metallindustrie eine Tariferhöhung von 5,0\%, ebenso ver.di für das Bankgewerbe. In den übrigen Branchen bewegten sich die Forderungen zwischen 4,0 und 4,5\%. Für die Deutsche Telekom strebte ver.di 6,0 \% an. Spitzenreiter war die Eisen- und Stahlindustrie. Hier forderte die IG Metall eine Lohnerhöhung von 7,0 \% (Übersicht 1).

\footnotetext{
Reinhard Bispinck, Dr., Wissenschaftler im WSI und Leiter des WSI-Tarifarchivs in der Hans-Böckler-Stiftung. e-mail:Reinhard-Bispinck@boeckler.de Götz Bauer, Monika Müller, Ulrich Schmidt, Monika Schwacke-Pilger, Andrea Taube und Monika Wiebel sind SachbearbeiterInnen im WSI-Tarifarchiv.
} 
Die Kündigungstermine der Vergütungstarifverträge lagen so, dass die Metallund Elektroindustrie die Tarifrunde eröffnete. Hier liefen die Lohn- und Gehaltstarifverträge Ende Februar 2006 aus. Ende März folgten Teile des Hotel- und Gaststättengewerbes und die Deutsche Telekom AG, Ende April Teile der Holz und Kunststoff verarbeitenden Industrie sowie die Textil- und Bekleidungsindustrie und Ende Mai das Bankgewerbe. Ende August war die Eisen- und Stahlindustrie an der Reihe.

Die ersten Abschlüsse des Jahres 2006 gab es in Tarifbereichen, in denen bereits seit vielen Monaten ergebnislos verhandelt worden war (Übersicht 2). Im Einzelhandel gelang ver.di erst Anfang Januar für Berlin ein Pilotabschluss für den Tarifbereich, in dem die Lohn- und Gehaltsverträge bereits Ende Juni 2005 ausgelaufen waren. Er sah nach sechs Nullmonaten eine Einmalzahlung von insgesamt $275 €$ für 2006/2007 und eine Tarifanhebung von $1,0 \% \mathrm{ab}$ 1.12.2006 bei einer Laufzeit bis Ende Juni 2007 vor. In der Papier verarbeitenden Industrie umfasste der Abschluss zwölf Nullmonate sowie eine Tarifsteigerung von 1,5 $\%$ ab dem 1.4.2006 und weitere $2,0 \%$ ab dem 1.4.2007 bei einer Laufzeit bis 31.3.2008. Im August 2006 erfolgte eine Einmalzahlung von $150 €$.

Der diese Tarifrunde prägende Abschluss kam in der Metall- und Elektroindustrie zustande. In Düsseldorf einigten sich die Tarifparteien am 22.4.2006 für Nordrhein-Westfalen in einem Pilotabschluss auf eine Pauschale von $310 €$ für März bis Mai 2006 sowie eine Tariferhöhung von 3,0 \% ab 1.6.2006. Die Laufzeit geht bis zum 31.3.2007. Dieser Abschluss war ein Referenzpunkt für die nachfolgenden Tarifverhandlungen anderer Branchen, der allerdings zumeist nicht vollständig erreicht wurde.

In der westdeutschen Textil- und Bekleidungsindustrie vereinbarten die Tarifparteien eine Pauschale von insgesamt $340 €$ für Mai bis Oktober 2006, gefolgt von einer Tariferhöhung von 2,5\% ab dem 1.11.2006 und einer Stufenerhöhung von 2,0 \% ab dem 1.5.2007 bei einer Laufzeit bis 29.2.2008.

Im Bankgewerbe (ohne Genossenschaftsbanken) vereinbarte ver.di einen zweistufigen Abschluss. Er sieht eine Pauschalzahlung von $100 €$ für Juni bis August sowie eine Anhebung der Tarifentgelte um 3,0 \% ab dem 1.9.2006 und weitere 1,5\% ab dem 1.12.2007 bis Juni 2008 vor.

\begin{tabular}{|lc|}
\hline $\begin{array}{l}\text { Übersicht 1: Tarifforderungen in der Tarifrunde } \\
\text { Tarifbereichen - in \% - }\end{array}$ \\
\hline Branche & $\begin{array}{c}\text { Lohn- und } \\
\text { Gehaltsforderung }\end{array}$ \\
\hline Bankgewerbe & 5,0 \\
Deutsche Post AG & 4,5 \\
Deutsche Telekom AG & 6,0 \\
Eisen- und Stahlindustrie & 7,0 \\
Energie- und Versorgungswirtschaft (AVEU) Ost & 5,9 \\
Holz und Kunststoff verarbeitende Industrie & 4,5 \\
Hotels und Gaststätten Nordrhein-Westfalen & 4,0 \\
Kfz-Gewerbe Hessen & 4,5 \\
Metallindustrie & 5,0 \\
Textil- und Bekleidungsindustrie West & 4,5 \\
\hline & VSI Hans Böckler \\
Quelle: WSI-Tarifarchiv. & Stiftung \\
\hline
\end{tabular}

\begin{tabular}{|c|c|c|}
\hline \multicolumn{3}{|c|}{$\begin{array}{l}\text { Übersicht 2: Ausgewählte Lohn- und Gehaltsabschlüsse West und Ost } \\
\text { für } 2006\end{array}$} \\
\hline Abschluss & Tarifbereich & Ergebnis \\
\hline 05.01 .2006 & $\begin{array}{l}\text { Einzelhandel Berlin } \\
\text { Pilotabschluss }\end{array}$ & $\begin{array}{l}\text { nach } 6 \text { Nullmonaten (Juli-Dezember 2005) } \\
200 / 75 € \text { Einmalzahlung für 2006/2007 } \\
1,0 \% \text { ab 01.12.06, Laufzeit bis 30.06.07 }\end{array}$ \\
\hline 17.02 .2006 & $\begin{array}{l}\text { Kfz-Gewerbe Nordrhein- } \\
\text { Westfalen }\end{array}$ & $\begin{array}{l}\text { nach } 10 \text { Nullmonaten (März-Dezember 2005) } \\
160 € \text { Pauschale insg. für Januar und Februar } 2006 \\
\mathbf{2 , 2} \% \text { ab 01.03.06, Laufzeit bis 28.02.07 }\end{array}$ \\
\hline 01.03 .2006 & $\begin{array}{l}\text { Papier verarbeitende } \\
\text { Industrie West }\end{array}$ & $\begin{array}{l}\text { nach } 12 \text { Nullmonaten (April 2005-März 2006) } \\
\mathbf{1 , 5} \% \text { ab 01.04.06, } 150 € \text { Einmalzahlung im August } 2006 \\
\mathbf{2 , 0} \% \text { Stufenerhöhung ab 01.04.07, Laufzeit bis } 31.03 .08\end{array}$ \\
\hline 20.03 .2006 & $\begin{array}{l}\text { Energie- und Versorgungs- } \\
\text { wirtschaft Ost (AVEU) }\end{array}$ & $\begin{array}{l}2,7 \% \text { ab } 01.03 .06 \\
200 € \text { zusätzliche Einmalzahlung für AN, die am } 01.03 .06 \\
\text { beschäftigt waren, Laufzeit bis } 31.03 .07\end{array}$ \\
\hline 11.04 .2006 & $\begin{array}{l}\text { Hotels und Gaststätten } \\
\text { Nordrhein-Westfalen }\end{array}$ & $\begin{array}{l}\text { nach einem Nullmonat (April 2006) } \\
1,5 \% \text { ab 01.05.06 } \\
1,4 \% \text { Stufenerhöhung ab 01.05.07, Laufzeit bis } 31.03 .08\end{array}$ \\
\hline 22.04 .2006 & $\begin{array}{l}\text { Metallindustrie Nordrhein- } \\
\text { Westfalen Pilotabschluss }\end{array}$ & $\begin{array}{l}310 € \text { Pauschale insg. für März-Mai } 2006 \\
3,0 \% \text { ab 01.06.06, Laufzeit bis } 31.03 .07\end{array}$ \\
\hline 12.05 .2006 & $\begin{array}{l}\text { Textil- und Bekleidungs- } \\
\text { industrie West }\end{array}$ & $\begin{array}{l}340 € \text { Pauschale insg. für Mai-Oktober } 2006 \\
2,5 \% \text { ab 01.11.06 } \\
2,0 \% \text { Stufenerhöhung ab 01.05.07, Laufzeit bis } 29.02 .08\end{array}$ \\
\hline 13.05 .2006 & Deutsche Post AG & $\begin{array}{l}250 € \text { Pauschale insg. für Mai-Oktober } 2006 \\
3,0 \% \text { ab 01.11.06 } \\
\mathbf{2 , 5} \% \text { Stufenerhöhung ab 01.11.07, Laufzeit bis } 30.04 .08\end{array}$ \\
\hline 17.05 .2006 & $\begin{array}{l}\text { Holz und Kunststoff } \\
\text { verarbeitende Industrie } \\
\text { Niedersachsen/Bremen }\end{array}$ & $\begin{array}{l}\text { nach einem Nullmonat (Mai 2006) } \\
350 € \text { Pauschale insg. für Juni-Oktober } 2006 \\
\mathbf{2 , 5} \% \text { ab 01.11.06, Laufzeit bis } 30.04 .07\end{array}$ \\
\hline 19.05.2006 & Öffentlicher Dienst Länder & $\begin{array}{l}\text { Pauschalzahlungen von } \\
150 / 100 / 50 € \text { im Juli } 2006 \\
310 / 210 / 60 € \text { im Januar } 2007 \\
450 / 300 / 100 € \text { im September } 2007 \\
\text { jew. für EntgGr. E } 1-8 / \mathrm{E} 9-12 / \mathrm{E} 13-15 \\
2,9 \% \text {, aufgerundet auf volle } 5 € \text { ab 01.01./01.05.08 } \\
\text { West/Ost, Laufzeit bis } 31.12 .08\end{array}$ \\
\hline 02.06 .2006 & Deutsche Telekom AG & $\begin{array}{l}350 € \text { Pauschale insg. für April-Oktober } 2006 \\
3,0 \% \text { ab 01.11.06, Laufzeit bis } 31.07 .07\end{array}$ \\
\hline 22.06 .2006 & $\begin{array}{l}\text { Bankgewerbe (ohne } \\
\text { Genossenschaftsbanken) }\end{array}$ & $\begin{array}{l}100 € \text { Pauschale insg. für Juni-August } 2006 \\
3,0 \% \text { ab 01.09.06 } \\
1,5 \% \text { Stufenerhöhung ab 01.12.07, Laufzeit bis } 30.06 .08\end{array}$ \\
\hline 04.09 .2006 & $\begin{array}{l}\text { Privates Verkehrsgewerbe } \\
\text { NRW }\end{array}$ & $\begin{array}{l}\text { nach } 4 \text { Nullmonaten (Mai-August) } \\
2,2 \% \text { ab } 01.09 .06 \\
1,8 \% \text { Stufenerhöhung ab } 01.08 .07 \text { bis } 30.04 .08\end{array}$ \\
\hline 21.09 .2006 & $\begin{array}{l}\text { Eisen- und Stahlindustrie } \\
\text { Niedersachsen, Bremen, } \\
\text { Nordrhein-Westfalen }\end{array}$ & $\begin{array}{l}500 € \text { Pauschale insg. für September-Dezember } 2006 \\
3,8 \% \text { ab } 01.01 .07,750 € \text { zusätzliche Einmalzahlung, } \\
\text { Laufzeit bis } 31.01 .08\end{array}$ \\
\hline 29.09 .2006 & Volkswagen AG & $\begin{array}{l}1.000 € \text { Pauschale insg. für Februar-Dezember } 2007 \text { bzw. } \\
\text { für ab dem 01.01.05 Beschäftigte } \\
3,0 \% \text { ab 01.02.07 } \\
\text { Übernahme des Tarifabschlusses Metall Niedersachsen } \\
2007 \text { ab 01.01. bis 01.02.08 }\end{array}$ \\
\hline & & WSI $\begin{array}{c}\text { Hans Böckler } \\
\text { Stiftung }\end{array}$ \\
\hline
\end{tabular}


Die beiden Abschlüsse bei der Deutschen Post AG und bei der Deutschen Telekom AG liegen am oberen Ende der Skala der Tarifrunde 2006. Die Beschäftigten der Deutschen Post AG erhalten für Mai bis Oktober $250 €$ Pauschale, ab dem 1.11.2006 eine Tariferhöhung von 3,0\% sowie eine Stufenerhöhung von $2,5 \%$ ab dem 1.11 . 2007 mit einer Laufzeit bis 30.4.2008. Bei der Deutschen Telekom AG sieht der Abschluss, der nach Schlichtung durch den CDU-Politiker Heiner Geißler zustande kam, eine Pauschalzahlung von $350 €$ für die Monate April bis Oktober sowie eine Tarifanhebung um 3,0 \% ab dem 1.11.2006 bis Ende Juli 2007 vor.

In der Eisen- und Stahlindustrie setzte die IG Metall am 21.9.2006 den wohl höchsten Branchenabschluss durch. Nach einer Pauschalzahlung von $500 €$ insgesamt für September bis Dezember 2006 erhalten die Beschäftigten eine Tarifsteigerung von 3,8 \% ab dem 1.1.2007 und eine zusätzliche Einmalzahlung von $750 €$. Der Vertrag hat eine Laufzeit bis Ende Januar 2008.

Bei der Volkswagen AG einigten sich die Tarifparteien am 29.9.2006 u.a. auf eine Beschäftigungs- und Arbeitsplatzsicherung durch verbindliche Produktions- und Auslastungszusagen sowie einen Arbeitszeitkorridor zwischen 25 und 33 Wochenstunden. Für Februar bis Dezember 2007 gibt es eine Einmalzahlung von $1.000 €$ bzw. für die ab 2005 eingestellten Beschäftigten eine Tariferhöhung von 3,0 \% ab dem 1.2.2007.

\subsection{KONFLIKT IM ÖFFENTLICHEN DIENST}

Im öffentlichen Dienst spielte sich ein vielschichtiger Tarifkonflikt ab, der nur schrittweise zu einem Ende kam. Die Kündigungen der Arbeitszeitbestimmungen des neuen Tarifvertrags für den öffentlichen Dienst (TVöD) durch die Kommunalen Arbeitgeberverbände in Hamburg, Niedersachsen und Baden-Württemberg führten zu streitigen Verhandlungen, die erst nach bis zu zweimonatigen Arbeitsniederlegungen beendet werden konnten. Für die Länder konnte ver.di - ebenfalls erst nach langwierigen Streiks - einen neuen Tarifvertrag (TV-L) abschließen, der sich in wichtigen Bestimmungen an den TVöD anlehnt. Die tariflichen Wochenarbeitszeiten wurden gegenüber dem TVöD bei den betroffenen Kommunen und bei den Ländern nach unterschiedlichen Kriterien differenziert an- gehoben. Sie bleiben im Durchschnitt zum Teil deutlich unter den von den Arbeitgebern geforderten 40 und mehr Wochenstunden. Die Länderbeschäftigten erhalten in den Jahren 2006 und 2007 drei nach Einkommen gestaffelte Pauschalzahlungen. Die neue Vergütungstabelle wird Anfang 2008 um 2,9 \% angehoben mit einer Laufzeit bis Ende des Jahres. Dem Marburger Bund gelang es nach längeren Arbeitskämpfen, sich auf Länder- und Kommunalebene als eigenständige Tarifvertragspartei für die KrankenhausärztInnen zu etablieren (ausführlich: Bispinck 2006).

\subsection{STREIKS UM SOZIALTARIF- VERTRÄGE}

In zahlreichen Unternehmen kam es im vergangenen Jahr zu Konflikten um Betriebsschließungen und Beschäftigungssicherung. Prominente Beispiele sind die Insolvenz des Handy-Herstellers BenQ (früher Siemens) oder auch die angekündigte Schließung ganzer Standorte bei der Allianz AG. In der Regel versuchen die Gewerkschaften in solchen Fällen, durch betriebliche Vereinbarungen Regelungen zu Beschäftigungsgesellschaften, Sozialplänen etc. durchzusetzen. Im vergangenen Jahr schlug die IG Metall in mehreren Unternehmen einen anderen Weg ein und versuchte erfolgreich, die Standorterhaltung, Beschäftigungssicherung bzw. soziale Absicherung bei Betriebsschließungen und Beschäftigungsabbau tarifvertraglich zu regeln und dies mit Hilfe von Arbeitskampfmaßnahmen durchzusetzen. Drei Streiks um Sozialtarifverträge fanden große öffentliche Aufmerksamkeit:

- Nach monatelangen Auseinandersetzungen um die Schließung des AEG-Werks in Nürnberg und einem mehr als sechswöchigen Streik einigten sich die IG Metall und Electrolux am 28.2.2006 auf einen Sozialtarifvertrag. Der Vertrag sieht neben sehr hohen Abfindungszahlungen für die betroffenen Beschäftigten auch die Einrichtung einer Qualifizierungs- und Beschäftigungsgesellschaft vor. Die SchlieBung des Standortes konnte nicht abgewendet werden, dennoch gilt dieser Sozialtarifvertrag bei AEG aus Sicht der IG Metall als ein großer Erfolg.

- In einem der längsten und härtesten Streiks in der Geschichte der Metallindustrie Berlins haben die Beschäftigten des Baumaschinenherstellers $\mathrm{CNH}$ einen So- zialplan und eine viermonatige Produktionsverlängerung bis Ende November 2006 erkämpft. Nach 107 Tagen Ausstand lenkte das Unternehmen Anfang Juni 2006 teilweise ein. Es stellte 29 Mio. $€$ für einen Sozialplan und für eine Beschäftigungsgesellschaft bereit. Die geplante Verlagerung der Produktion konnte nicht verhindert werden.

- Nach drei Wochen Streik, zahlreichen Aktionen und zähen Auseinandersetzungen führten Verhandlungen zwischen IG Metall, dem Verband der Metall- und Elektroindustrie (VME) und Bosch-SiemensHausgeräte (BSH) in Berlin am 18.10. zu einer Einigung: Der Beschluss zur SchlieBung des Produktionsstandortes in BerlinGartenfeld wurde zurückgenommen, die Fertigung allerdings auf rund die Hälfte reduziert; ca. 270 von 570 Arbeitsplätzen blieben erhalten. Für weitere 130 Beschäftigte konnten die Arbeitsverhältnisse ebenfalls gesichert werden. Für diese insgesamt 400 ArbeitnehmerInnen gilt eine Beschäftigungssicherung bis zum 31.7.2010.

Verlauf und Ergebnisse dieser Konflikte, die hier nicht im Einzelnen analysiert werden können, waren durchaus unterschiedlich. Gemeinsam ist ihnen allerdings der Ansatz, unter bestimmten Voraussetzungen die Auseinandersetzung um die Sicherung von Standorten und Arbeitsplätzen konflikt- und streikfähig zu machen (Seebacher 2006). Das Instrument des Streiks um Sozialtarifverträge ist in der Folge auch zum Gegenstand heftiger Kritik der Arbeitgeberverbände geworden. So bezeichnete Gesamtmetall-Präsident Kannegießer diese „Vermischung von Tarifautonomie und Betriebsverfassung“ als eine „böse Sackgasse“ und insgesamt „höchst fragwürdig“ (Osnabrücker Zeitung vom 4.3.2006). Die Bundesvereinigung der Deutschen Arbeitgeberverbände (BDA) erklärte Arbeitskämpfe für Tarifsozialpläne in Firmen, die Mitglied in einem Arbeitgeberverband sind, für rechtswidrig und forderte eine entsprechende gesetzliche Klarstellung (BDA 2006, S. 46 f.). Diese Auffassung wurde u. a. durch das Hessische Landesarbeitsgericht (Urteil vom 2. Februar 2006) zurückgewiesen.

\subsection{BEWERTUNG}

Die Tarifrunde 2006 zeigte aus Sicht der Arbeitgeber „ein geteiltes Bild“ (BDA 2006a, S. 26). Die BDA begrüßte, dass zu- 
mindest zu Beginn des Jahres an die „moderate Lohnentwicklung der letzten Jahre" angeknüpft wurde. Die höheren Abschlüsse seien auch Ausdruck der gesamtwirtschaftlichen Erholung in diesem Jahr. Für die Unternehmen seien durch neue Öffnungsklauseln, insbesondere durch betrieblich abdingbare Einmalzahlungen, „zusätzliche Gestaltungsspielräume“ entstanden. Dadurch sei die "neue Balance“ zwischen Flächentarifvertrag und betrieblichen Gestaltungsmöglichkeiten „weiter ausgebaut" worden (BDA 2006b, S. 38).

Der Sachverständigenrat sprach ebenfalls von einem „moderaten Zuwachs“ der Tariflöhne (SVR 2006a, Ziff. 93). Die Tarifvertragsparteien hätten in den vergangenen Jahren den Verteilungsspielraum nicht ausgeschöpft und insofern sei es gerechtfertigt, von einer „beschäftigungsfreundlichen Lohnpolitik" zu sprechen. Allerdings bedürfe es ergänzend einer weiteren „Auffächerung der Lohnstruktur" (ebenda, Ziff. 490f.), insbesondere im unteren Lohnsegment.

Das Ratsmitglied Peter Bofinger widersprach dieser Auffassung in einem Minderheitsvotum. Er machte darauf aufmerksam, dass man aus der Zunahme sozialversicherungspflichtiger Beschäftigung bei einem kräftigen Wirtschaftswachstum keinen besonderen Erfolg der Arbeitsmarktreformen oder der Lohnzurückhaltung der letzten Jahre ablesen könne (ebenda, Ziff. 554).

\section{Tarifergebnisse in Zahlen und Fakten}

\subsection{LOHN UND GEHALT}

Im Jahr 2006 wurden - wie bereits im Vorjahr - nur für einen Teil der Beschäftigten neue Lohn- und Gehaltstarifverträge abgeschlossen, weil wegen der länger laufenden Vergütungsabkommen aus dem Jahr 2005 eine ganze Reihe von Wirtschaftszweigen nicht an der Tarifrunde teilnahm. Dazu gehörten u.a. die Bereiche Druckindustrie, Bauhauptgewerbe, Versicherungsgewerbe, Groß- und Außenhandel und der öffentliche Dienst (Bund, Gemeinden).

Die DGB-Gewerkschaften schlossen 2006 in ganz Deutschland Lohn- und Gehaltstarifverträge für 9,7 Mio. Beschäftigte $\mathrm{ab}$, davon für 8,3 Mio. in den alten und

Tabelle 1: Laufzeit der Tarifverträge - in Monaten -

\begin{tabular}{|lllllllllllll|} 
& 1995 & $\mathbf{1 9 9 6}$ & $\mathbf{1 9 9 7}$ & $\mathbf{1 9 9 8}$ & $\mathbf{1 9 9 9}$ & $\mathbf{2 0 0 0}$ & $\mathbf{2 0 0 1}$ & $\mathbf{2 0 0 2}$ & $\mathbf{2 0 0 3}$ & $\mathbf{2 0 0 4}$ & $\mathbf{2 0 0 5}$ & $\mathbf{2 0 0 6}$ \\
\hline West & 15,1 & 16,2 & 16,8 & 12,7 & 13,8 & 21,5 & 14,1 & 18,1 & 20,4 & 21,8 & 25,2 & 21,6 \\
\hline Ost & & & & 14,7 & 23,3 & 16,4 & 19,7 & 21,0 & 22,0 & 28,4 & 24,7 \\
\hline \\
Quelle: WSI-Tarifarchiv, Stand: 31.12 .2006$. & & & & & & WSI & $\begin{array}{c}\text { Hans Böckler } \\
\text { Stiftung ale }\end{array}$
\end{tabular}

1,4 Mio. in den neuen Bundesländern. Das entspricht rund $51 \%$ der von Tarifverträgen erfassten Beschäftigten. Für weitere 4,2 Mio. Beschäftigte traten Erhöhungen in Kraft, die bereits 2005 oder früher vereinbart worden waren. Bei rund 5 Mio. Beschäftigten liefen 2006 oder früher die Vergütungstarifverträge aus, aber es kam bis zum Jahresende (noch) nicht zu Neuabschlüssen.

Die tarifliche Abschlussrate belief sich gesamtwirtschaftlich im Durchschnitt auf 2,6\% (2005: 1,8\%), in Westdeutschland betrug die Rate 2,7 \%, in Ostdeutschland 2,5\%. Die Abschlussrate schließt alle, gegebenenfalls auch 2007 und später in Kraft tretenden tabellenwirksamen Erhöhungen ein. Nicht berücksichtigt werden dagegen Pauschalzahlungen und zusätzliche Einmalzahlungen, die sich nicht dauerhaft in den Tariftabellen niederschlagen. Die Spannweite der durchschnittlichen Gesamtabschlussraten reicht von $1,0 \%$ im Wirtschaftsbereich Handel über 1,6 \% im Bereich Private Dienstleistungen, Organisationen ohne Erwerbszweck, 2,9 \% im Gartenbau, Land- und Forstwirtschaft bis hin $\mathrm{zu}$ 4,6\% im Kredit- und Versicherungsgewerbe. Diese Gesamtabschlussraten sind insofern von begrenzter Aussagekraft, weil sie sich immer auf die gesamte, je nach Tarifbereich sehr unterschiedlich lange Laufzeit der Tarifabkommen beziehen.

Betrachtet man lediglich die im Jahr 2006 in Kraft getretenen Tariferhöhungen, ergibt sich für West- und Ostdeutschland eine Abschlussrate von 2,3\%. Differenziert man diese Größe nach Wirtschaftsbereichen, dann zeigt sich für 2006 eine Streuung zwischen 1,0 \% und 3,0\%. Dabei ist allerdings zu berücksichtigen, dass die Erhöhungen zu unterschiedlichen Zeitpunkten im Jahresverlauf wirksam wurden.

Wie bereits in den Vorjahren spielten auch im Jahr 2006 „Nullmonate” bei den Tarifabschlüssen eine bedeutende Rolle. Für rund 8,8 Mio. (2005: 5,9 Mio.), das entspricht rund $90 \%$ der von Neuabschlüssen begünstigten Beschäftigten, gab es Tarifabschlüsse mit verzögerter Anpassung der
Lohn- und Gehaltserhöhungen. Von denen mussten knapp $48 \%$ zwischen einem Monat bis drei Monate auf die reguläre Tariferhöhung warten, für weitere knapp $6 \%$ vergingen vier bis fünf Monate bis zur ersten Tarifsteigerung, rund $46 \%$ mussten sogar sechs und mehr Nullmonate akzeptieren. Als Ausgleich vereinbarten die Gewerkschaften für die meisten $(87 \%)$ der davon betroffenen Beschäftigten Pauschalzahlungen, die durchschnittlich $60 €$ (West: $61 €$, Ost: $48 €)$ im Monat betrugen.

\section{TARIFDATEN 2006}

Der seit einigen Jahren zu beobachtende Trend zu längeren Laufzeiten hat sich im vergangenen Jahr leicht abgeschwächt fortgesetzt (Tabelle 1). Die Laufzeit der Vergütungstarifverträge im Jahr 2006 beträgt durchschnittlich 22,1 Monate (2005: 25,7 Monate). Für rund 4 Mio. Beschäftigte (42\%) laufen die Abkommen zwischen elf und 13 Monaten, für 4,9 Mio. (51\%) 24 Monate und länger, der Rest verteilt sich auf unterschiedliche Laufzeiten. In den neuen Bundesländern fällt die Laufzeit mit durchschnittlich 24,7 Monaten länger aus als im Westen mit 21,6 Monaten.

Bei der jahresbezogenen Steigerung der tariflichen Grundlöhne und -gehälter werden im Unterschied zur tariflichen Abschlussrate die Auswirkungen aus der (oft unterschiedlichen) Lage und Laufzeit der Tarifabkommen berücksichtigt. Auch werden gegebenenfalls im Berichtsjahr wirksam werdende Abschlüsse aus den Vorjahren sowie zusätzliche Einmalzahlungen und Pauschalzahlungen als Ausgleich für Abschlussverzögerungen mit einbezogen. Die jahresbezogene Tarifsteigerung setzt die durchschnittliche tarifliche Grundvergütung des gesamten Jahres 2006 zum Vorjahr in Bezug und erfasst insgesamt 15,7 Mio. ArbeitnehmerInnen. Diese kalenderjährliche Steigerung der Tarifverdienste 2006 gegenüber 2005 betrug für ganz Deutschland 1,5 \% (Tabelle 2). Am höchsten fiel die jahresbezogene Tarifsteigerung mit 2,7 \% im Bereich des Grundstoff- und Produktionsgütergewerbes aus, gefolgt vom Investitionsgütergewerbe mit 2,4 \%. 
Tabelle 2: Tarifsteigerung 20061 - in \% -

\begin{tabular}{lccc} 
Wirtschaftsbereich & Ost & West & Gesamt \\
\hline Gartenbau, Land- und Forstwirtschaft & 1,4 & 1,1 & 1,2 \\
Energie- und Wasserversorgung, Bergbau & 2,0 & 1,8 & 1,8 \\
Grundstoff- und Produktionsgütergewerbe & 3,3 & 2,6 & 2,7 \\
Investitionsgütergewerbe & 2,2 & 2,4 & 2,4 \\
Verbrauchsgütergewerbe & 1,5 & 1,5 & 1,5 \\
Nahrungs- und Genussmittelgewerbe & 2,2 & 1,5 & 1,6 \\
Baugewerbe & 0,8 & 0,6 & 0,7 \\
Handel & 0,7 & 0,9 & 0,9 \\
Verkehr und Nachrichtenübermittlung & 3,1 & 1,8 & 2,0 \\
Kreditinstitute, Versicherungsgewerbe & 2,1 & 2,1 & 2,1 \\
Priv. Dienstleistungen, Organ. o. Erwerbszweck & 1,1 & 0,8 & 0,8 \\
Gebietskörperschaften, Sozialversicherung & 0,9 & 0,3 & 0,5 \\
\hline Gesamte Wirtschaft & $\mathbf{1 , 4}$ & $\mathbf{1 , 5}$ & $\mathbf{1 , 5}$ \\
\hline 1) Jahresbezogene Erhöhung der tariflichen Grundvergütung 2006 gegenüber 2005. & W/SI Hans Böckler \\
Quelle: WSI-Tarifarchiv, Stand: 31.12.2006. & & & Stiftung
\end{tabular}

Tabelle 3: Tarifniveau Ost/West - in \% -

\begin{tabular}{|ccccccccccc|}
\hline 1996 & 1997 & 1998 & 1999 & 2000 & 2001 & 2002 & 2003 & 2004 & 2005 & 2006 \\
\hline 88,7 & 89,8 & 90,8 & 91,5 & 91,9 & 92,3 & 92,8 & 93,4 & 94,0 & 94,6 & 95,1 \\
\hline \\
Quelle: WSI-Tarifarchiv, Stand: 31.12 .2006$.
\end{tabular}

Am niedrigsten fiel die Steigerung im Bereich Gebietskörperschaften, Sozialversicherung mit 0,5\% aus. Ebenfalls relativ niedrig sind die Werte für das Baugewerbe $(0,7 \%)$, den Bereich Private Dienstleistungen, Organisationen ohne Erwerbszweck $(0,8 \%)$, den Handel $(0,9 \%)$ und den Bereich Gartenbau, Land- und Forstwirtschaft (1,2\%). Zwischen West- und Ostdeutschland ergaben sich für 2006 bei den Gesamtwerten kaum Unterschiede (West: $1,5 \%$, Ost: $1,4 \%$ ).

Der Stand der tariflichen Lohnangleichung an das Westniveau kann an der Entwicklung der tariflichen Grundvergütung festgemacht werden. Für den Stichtag 31.12.2006 ergibt sich dabei folgendes Bild (Tabelle 3): Auf Basis von knapp 40 Tarifbereichen/-branchen mit 1,8 Mio. erfassten Beschäftigten errechnet sich ein durchschnittliches Tarifniveau von 95,1\% (2005: 94,6\%). Der Anstieg ist auf Anpassungen u.a. in den Bereichen chemische Industrie und Energiewirtschaft zurückzuführen. Tabelle 3 zeigt, dass das Tempo der tariflichen Anpassung seit Jahren sehr gering ist. Seit dem Jahr 2002 steigt das Tarifniveau jährlich um rund 0,6 Prozentpunkte. Für wenige Tarifbereiche sind weitere Tarifan- hebungen vereinbart. Dazu gehören die chemische Industrie und der öffentliche Dienst.

Die Steigerung der Ausbildungsvergütungen ist auch im vergangenen Jahr wiederum sehr gering ausgefallen. Nach Berechnungen des Bundesinstituts für Berufsbildung ergibt sich ein Anstieg von lediglich 1,1\% (West: 1,0\%, Ost: 1,3\%), der damit noch deutlich unter der Steigerung der tariflichen Grundvergütungen liegt (BIBB 2007). Je nach Tarifbereich verbergen sich hinter diesen Durchschnittszahlen große Unterschiede: Gemessen an der Ausbildungsvergütung im dritten Ausbildungsjahr lagen die Beträge Ende 2006 in immerhin 11 bzw. 9 (West/Ost) der ausgewählten Tarifbereiche unverändert auf dem Vorjahresniveau. Im Übrigen variierten die Anhebungen zwischen 0,9\% z. B. im KfzGewerbe und $4,4 \%$ in der bayerischen Bekleidungsindustrie.

\section{TARIF- UND EFFEKTIVLOHNENT- WICKLUNG}

Die durchschnittliche jahresbezogene Tater dem kostenneutralen Verteilungsspielrifsteigerung 2006 von 1,5\% lag erneut un- raum aus Preissteigerung $(+1,7 \%)$ und Produktivitätszuwachs $(+1,9 \%$ je Arbeitsstunde). Die tariflichen Vergütungen sanken real um $0,2 \%$.

Betrachtet man die Effektiveinkommensentwicklung in Gesamtdeutschland, so ergibt sich folgendes Bild (vgl. Statistisches Bundesamt 2007): Die Summe der Bruttolöhne und -gehälter stieg 2006 um $1,4 \%$. Je beschäftigte/n Arbeitnehmer/in ergibt sich ein Anstieg um 0,7\%. Dies zeigt, dass der seit Jahren anhaltende Trend einer ausgeprägten negativen Lohndrift sich auch in 2006 fortgesetzt hat (Tabelle 4). Darin kommen mehrere Faktoren zum Ausdruck: Die Tarifbindung ist nach jüngsten Zahlen weiterhin leicht rückläufig, sodass ein wachsender Anteil der Branchen und Betriebe nicht erfasst wird. Tariflose Zustände in manchen Wirtschaftszweigen, die zunehmende Zahl von tariflichen Öffnungsklauseln, der weitere Abbau übertariflicher Leistungen und auch der wachsende Lohndruck durch die Arbeitsmarktreformen trugen maßgeblich zum Auseinanderklaffen von tariflicher und effektiver Einkommensentwicklung bei. Außerdem wirkt sich auch die steigende Zahl von Teilzeitbeschäftigungsverhältnissen (inkl. Mini-Jobs) senkend auf das durchschnittliche effektive Bruttoeinkommen aus.

Die Lohnstückkosten gingen 2006 erneut zurück - und zwar um 1,1 \% nach $0,3 \%$ im Vorjahr. Im internationalen Vergleich blieb es bei dem bekannten Bild: In allen wichtigen Konkurrenzländern innerhalb der EU und auch in den USA wuchsen die Lohnstückkosten erheblich stärker.

\subsection{ARBEITSZEIT}

Die gesamtwirtschaftlichen Eckdaten der tariflichen Wochenarbeitszeit sind im Laufe des vergangenen Jahres weitgehend gleich geblieben, auch wenn sich die tariflichen Arbeitszeitstandards in einzelnen Tarifbereichen geändert haben. ${ }^{1}$ So wurde die tarifliche Wochenarbeitszeit $u$. a. in einigen Bereichen des öffentlichen Dienstes heraufgesetzt.

Die tarifliche Wochenarbeitszeit betrug Ende 2006 im gesamtwirtschaftlichen
Tabelle 4: Lohndrift gegenüber dem Vorjahr ${ }^{1}$ - in \% -

\begin{tabular}{|llllllllllll|}
\hline 1995 & 1996 & 1997 & 1998 & 1999 & 2000 & 2001 & 2002 & 2003 & 2004 & 2005 & 20062 \\
\hline$-1,4$ & $-1,0$ & $-1,3$ & $-0,9$ & $-1,4$ & $-0,5$ & $-0,1$ & $-1,3$ & $-0,8$ & $-0,7$ & $-0,9$ & $-0,6$ \\
\hline $\begin{array}{l}\text { 1) Abweichung der Löhne und Gehälter je Arbeitnehmerln von den Tarifverdiensten auf Monatsbasis. } \\
\text { 2) Werte für die ersten drei Quartale 2006. } \\
\text { Quelle: Deutsche Bundesbank 2006. }\end{array}$ \\
W
\end{tabular}

Auswirkungen auf die durchschnittliche tarifliche Wochenarbeitszeit haben im Übrigen auch Veränderungen der Beschäftigtenzahlen, mit denen die Arbeitszeitwerte der Tarifbereiche gewichtet werden. 
Durchschnitt in ganz Deutschland 37,7 Stunden (West: 37,4 und Ost: 38,9 Stunden) (Übersicht 3). Angesichts der zahlreichen arbeitszeitbezogenen Öffnungsklauseln und Flexi-Bestimmungen (u. a. mit Kontenregelungen und langen Ausgleichszeiträumen) müssen die gesamtwirtschaftlichen und branchenbezogenen Zahlen zur tariflichen Arbeitszeitdauer eher als Referenzgrößen denn als Beschreibung der tatsächlichen Arbeitszeitstandards angesehen werden.

Die tarifliche Urlaubsdauer (Endstufe) beträgt im gesamtdeutschen Durchschnitt unverändert 30,0 Tage (West: 30,1 und Ost: 29,5 Tage). Errechnet man auf Basis dieser und weiterer Einzelkomponenten die tarifliche Jahresarbeitszeit, so ergibt sich ein gesamtdeutscher Durchschnitt von 1.657,0 Stunden, für Westdeutschland 1.644,7 und für Ostdeutschland 1.720,3 Stunden (Tabelle 5).

\section{3 \\ Allgemeinverbindlichkeit, Entsendegesetz, Kombi- und Mindestlohn}

Die politische Auseinandersetzung um die bessere Sicherung von Mindeststandards für Arbeits- und Einkommensbedingungen wurde auch im vergangenen Jahr weitergeführt. Nach der Bundestagswahl im Herbst 2005 hatte die Große Koalition aus CDU/CSU und SPD vereinbart, ein „Kombi-Lohn-Modell“ zu prüfen. Dabei soll sichergestellt werden, dass die „Löhne nicht in den Bereich der Sittenwidrigkeit heruntergedrückt" werden können, aber andererseits Menschen „mehr als bisher die Möglichkeit auch zur Beschäftigung mit niedrigen Einkommen" erhalten. Auch die Bezüge zu den Themen Entsendegesetz und Mindestlohn sowie die Auswirkungen der EU-Dienstleistungsrichtlinie sollen in diesem Zusammenhang berücksichtigt werden (Koalitionsvertrag 2005, S. 24f.). Bundesarbeitsminister Franz Müntefering hatte zunächst für den Herbst ein Gesamtkonzept zu diesem Komplex angekündigt, jedoch ist es weder dazu noch zu einer politischen Entscheidung der Regierung oder des Gesetzgebers gekommen. Ende Januar 2007 sollte ein entsprechender Vorschlag unterbreitet werden.

\begin{tabular}{|c|c|c|c|}
\hline \multicolumn{4}{|c|}{ Übersicht 3: Tarifliche Arbeitszeitregelungen 2006} \\
\hline Tarifregelung & Ost & West & Gesamt \\
\hline Wochenarbeitszeit (Std.) & 38,9 & 37,4 & 37,7 \\
\hline Anteil der Beschäftigten - in \% - mit: & & & \\
\hline bis zu 35 Std. & 3,5 & 24,1 & 20,7 \\
\hline 36-37 Std. & 5,6 & 10,5 & 9,7 \\
\hline $37,5-38,5$ Std. & 30,7 & 39,9 & 38,3 \\
\hline 39-40 Std. und mehr & 60,1 & 25,2 & 30,9 \\
\hline Urlaub (Arbeitstage) ${ }^{1}$ & 29,5 & 30,1 & 30,0 \\
\hline Jahresarbeitszeit (Std.) & $1.720,3$ & $1.644,7$ & $1.657,0$ \\
\hline $\begin{array}{l}\text { 1) Endstufe } \\
\text { Quelle: WSI-Tarifarchiv, Stand: } 31.12 .2006 .\end{array}$ & & & $\begin{array}{l}\text { Hans Böckler } \\
\text { Stiftung }\end{array}$ \\
\hline
\end{tabular}

Tabelle 5: Tarifliche Wochen- und Jahresarbeitszeit 1997-2006 - in Std. -

\begin{tabular}{|c|c|c|c|c|c|c|c|c|c|c|c|}
\hline & & 1997 & 1998 & 1999 & 2000 & 2001 & 2002 & 2003 & 2004 & 2005 & 2006 \\
\hline \multirow[t]{2}{*}{ Woche } & W & 37,5 & 37,4 & 37,4 & 37,4 & 37,4 & 37,4 & 37,4 & 37,4 & 37,4 & 37,4 \\
\hline & O & 39,4 & 39,4 & 39,2 & 39,1 & 39,1 & 39,1 & 39,0 & 38,9 & 39,0 & 38,9 \\
\hline \multirow[t]{2}{*}{ Jahr } & W & 1644,4 & 1643,2 & 1642,8 & 1642,5 & 1641,9 & 1642,6 & 1643,5 & 1643,3 & 1643,2 & 1644,7 \\
\hline & 0 & - & 1735,5 & 1729,9 & 1727,7 & 1724,2 & 1722,7 & 1721,9 & 1719,2 & 1718,9 & 1720,3 \\
\hline
\end{tabular}

\section{ALLGEMEINVERBINDLICH- ERKLÄRUNG UND ARBEITNEHMER- ENTSENDEGESETZ}

Das Instrument der Allgemeinverbindlicherklärung (AVE) hat in Deutschland auch im internationalen Vergleich - nur eine geringe Bedeutung. Überdies hat sich der rückläufige Trend bei der Nutzung von Allgemeinverbindlicherklärungen auch im vergangenen Jahr fortgesetzt. Anfang 2006 waren nur noch 446 Tarifverträge allgemeinverbindlich erklärt (Tabelle 6), darunter 232 Ursprungs- und 214 Änderungsund Ergänzungstarifverträge. Dies bedeutet seit Mitte der 1990er Jahre einen Rückgang um rund $30 \%$. Bezogen auf die gültigen Ursprungstarifverträge sind heute nur rund 1,5\% der Tarifverträge allgemeinverbindlich.

Lediglich eine sehr geringe Zahl der allgemeinverbindlichen Tarifverträge bezieht sich direkt auf die Tariflöhne und -gehälter. Dies betrifft bei der klassischen AVE im Wesentlichen das Friseurgewerbe, das Wach- und Sicherheitsgewerbe und das Gebäudereinigerhandwerk. Hinzu kommen die über das Arbeitnehmer-Entsendegesetz (AEntG) allgemeinverbindlich erklärten Tarifverträge über Mindestlöhne im Bauhauptgewerbe, im Abbruchgewerbe, im Maler- und Lackiererhandwerk sowie im Dachdeckerhandwerk. Die in diesen Branchen festgelegten Mindestlöhne variieren zwischen 7,15 und $12,50 €(\ddot{U}$ bersicht 4).

\begin{tabular}{|c|c|c|c|}
\hline \multicolumn{4}{|c|}{$\begin{array}{l}\text { Tabelle 6: Allgemeinverbind- } \\
\text { liche Tarifverträge 1995-2006 }\end{array}$} \\
\hline Jahr* & Gesamt & $\mathrm{Neu}$ & Außer Kraft \\
\hline 1995 & 627 & 136 & 192 \\
\hline 1996 & 571 & 145 & 158 \\
\hline 1997 & 558 & 140 & 110 \\
\hline 1998 & 588 & 99 & 96 \\
\hline 1999 & 591 & 102 & 142 \\
\hline 2000 & 551 & 82 & 99 \\
\hline 2001 & 534 & 59 & 51 \\
\hline 2002 & 542 & 56 & 118 \\
\hline 2003 & 480 & 50 & 54 \\
\hline 2004 & 476 & 37 & 37 \\
\hline 2005 & 475 & 19 & 30 \\
\hline 2006 & 446 & 16 & 54 \\
\hline
\end{tabular}

Ein tariflicher Mindestlohn wurde auch für den Bereich der Zeitarbeit/Leiharbeit vereinbart. Nach langwierigen Verhandlungen einigte sich die DGB-Tarifgemeinschaft Zeitarbeit mit dem Bundesverband Zeitarbeit/Personaldienstleistungen (BZA) und dem Interessenverband Deutscher Zeitarbeitsunternehmen (iGZ) auf einen Tarifvertrag zur Regelung von Mindestarbeitsbedingungen für die Zeitarbeitsbranche. Als Mindestentgelt wurde ein Stundenlohn von 7,00€ (West) und 6,10 $€$ (Ost) ab Juli 2006 vereinbart. Dieser Mindestlohn soll 2007 auf $7,15 €(6,22 €$ Ost) und in 2008 auf $7,31 €(6,36 €$ Ost) steigen. Zusätzlich wurden Regelungen zum Mindesturlaub und zum Urlaubsgeld getroffen. Dieser Tarifvertrag sollte zum 


\begin{tabular}{|c|c|c|c|c|}
\hline \multicolumn{5}{|c|}{$\begin{array}{l}\text { Übersicht 4: Tarifliche Mindestlöhne nach dem Arbeitnehmer- } \\
\text { Entsendegesetz - in €- }\end{array}$} \\
\hline Tarifgebiet & Lohngruppe & & & \\
\hline Bauhauptgewerbe & & ab 1.9.06 & ab 1.9.07 & \\
\hline \multirow[t]{2}{*}{ Bundesgebiet West } & 1 Werker & 10,30 & 10,40 & \\
\hline & 2 Fachwerker & 12,40 & 12,50 & \\
\hline \multirow[t]{2}{*}{ Bundesgebiet Ost } & 1 Werker & 8,90 & 9,00 & \\
\hline & 2 Fachwerker & 9,80 & 9,80 & \\
\hline Dachdeckerhandwerk & & ab 1.1 .06 & ab 1.1.08 & ab 1.1.09 \\
\hline West und Ost & Helfer & 10,00 & 10,20 & 10,40 \\
\hline Maler- und Lackierhandwerk & & ab 1.4.05 & & \\
\hline \multirow[t]{2}{*}{ West } & ungelernter AN & 7,85 & & \\
\hline & Gesellen & 10,73 & & \\
\hline \multirow[t]{2}{*}{ Ost } & ungelernter $\mathrm{AN}$ & 7,15 & & \\
\hline & Gesellen & 9,37 & & \\
\hline Abbruch- u. Abwrackgewerbe & & ab 1.9.05 & & \\
\hline \multirow[t]{2}{*}{ West } & Hilfskraft & 9,49 & & \\
\hline & Fachwerker & 11,60 & & \\
\hline \multirow[t]{2}{*}{ Ost } & Hilfskraft & 8,80 & & \\
\hline & Fachwerker & 9,80 & & \\
\hline Quelle: WSI-Tarifarchiv, Stand: 31.12.2006. & & & W & $\begin{array}{l}\text { Hans Böckle } \\
\text { Stiftung }\end{array}$ \\
\hline
\end{tabular}

1.7.2006, frühestens jedoch mit dem Erlass einer Rechtsverordnung zur Allgemeinverbindlicherklärung durch den Bundesminister für Arbeit und Soziales, in Kraft treten. Voraussetzung dafür ist die Erweiterung des Arbeitnehmer-Entsendegesetzes auf die Zeitarbeitsbranche.

\section{KOMBILÖHNE - MINDESTLOHN}

Die Fronten in der Debatte haben sich nicht fundamental verschoben. In der Bundesregierung blieb die Skepsis gegenüber flächendeckenden Modellen für einen Kombilohn nach wie vor groß. Meinungsverschiedenheiten bestanden weiterhin in der Frage, ob und wie durch zielgruppenspezifische Maßnahmen die Eingliederung von Gruppen mit besonderen Vermittlungsproblemen erleichtert werden könnte. In Sachen einheitlicher gesetzlicher Mindestlohn blieb die Union auf ihrem strikt ablehnenden Kurs. Innerhalb der SPD gab es dazu unterschiedliche Positionen. In einem Papier des SPD-Gewerkschaftsrates vom 21.9.2006 wird ein Konzept mit mehreren Elementen formuliert. Danach soll der Geltungsbereich des Arbeitnehmer-Entsendegesetzes auf alle Wirtschaftsbereiche ausgeweitet werden, um hierdurch branchenbezogene Mindestlöhne zu ermöglichen. Um die Blockadehaltung der Bundesvereinigung der Deutschen Arbeitgeberverbände (BDA) und vieler Arbeitgeberverbände bei der Anwendung der Allgemeinverbindlicherklärung nach dem Tarifvertragsgesetz aufzubrechen, sollen (gegebenenfalls außerhalb des
Tarifvertragsgesetzes) Regelungen geschaffen werden, die eine Allgemeinverbindlicherklärung leichter ermöglichen. Schließlich soll für Branchen, in denen es keine Tarifverträge gibt oder diese nicht greifen, sowie für Branchen, in denen die Tarifentgelte ein bestimmtes Mindestniveau unterschreiten, ein einheitlicher gesetzlicher Mindestlohn eingeführt werden. Die Höhe soll vergleichbar zum Niveau der wichtigsten europäischen Nachbarländer festgelegt werden (Tabelle 7).

Die Kombilohndebatte wurde im Wesentlichen von zwei Modellen beeinflusst. Der Sachverständigenrat erstellte im Auftrag der Bundesregierung ein Gutachten, das im Kern vorsieht, den Anspruch auf Unterstützungsleistungen in Höhe des bisherigen Arbeitslosengeldes II (ALG II) von einer Gegenleistung abhängig zu machen (SVR 2006b). Die Anreize für die Empfänger von ALG II zur Aufnahme einer re-

\begin{tabular}{|c|c|c|}
\hline \multicolumn{3}{|c|}{$\begin{array}{l}\text { Tabelle 7: Gesetzliche Mindest- } \\
\text { löhne in Europa } 2007\end{array}$} \\
\hline Land & Euro/Stunde & $\begin{array}{l}\text { Anstieg } \\
2006 / 2007 \\
- \text { in } \%-\end{array}$ \\
\hline Luxemburg & 9,08 & 4,5 \\
\hline Irland & 8,30 & 8,5 \\
\hline Frankreich & 8,27 & 3,0 \\
\hline Niederlande & 8,13 & 5,7 \\
\hline Großbritannien & 7,96 & 8,2 \\
\hline Belgien & 7,93 & 1,9 \\
\hline DGB-Forderung & 7,50 & \\
\hline $\begin{array}{l}\text { Quelle: Eurostat; } \\
\text { Berechnungen des WSI, } \\
\text { Stand: Januar } 2007 .\end{array}$ & WSI & $\begin{array}{l}\text { Hans Böckler } \\
\text { Stiftung }\end{array}$ \\
\hline
\end{tabular}
gulären Arbeit sollen dahingehend ver- stärkt werden, dass bei einer Tätigkeit auf dem ersten Arbeitsmarkt die Hälfte des Hinzuverdienstes (statt wie bisher ein Fünftel) beim Leistungsempfänger verbleibt. Im Gegenzug erfolgt allerdings eine Absenkung des ALG II-Regelsatzes für erwerbsfähige Leistungsempfänger in Höhe von $30 \%$ (SVR 2006a, Ziff. 513ff.).

Der zweite Vorschlag stammt vom SVR-Mitglied Peter Bofinger und dem IABVizedirektor Ulrich Walwei. Sie schlugen ein am ALG II-Anspruch orientiertes abgabenfreies Mindesteinkommen bei Vollzeitbeschäftigung in Höhe von $750 €$ für Singles und $1.300 €$ für Paare vor. Die Umsetzung soll durch eine bedarfsorientierte Steuergutschrift erfolgen. Ergänzend soll ein Mindestlohn von 4,50€ je Stunde bei Beibehaltung des Regelsatzes für ALG IIEmpfänger eingeführt werden (Bofinger et al. 2006).

Von gewerkschaftlicher Seite wurde insbesondere das SVR-Modell abgelehnt, aber auch das Bofinger/Walwei-Modell, das bei der SPD durchaus Anklang fand (SPD 2007) wurde teilweise sehr kritisch kommentiert. In einer Stellungnahme von ver.di wird zwar anerkannt, dass die problematische Minijob-Subventionierung nach diesem Modell künftig entfallen soll, zugleich aber wird die „,noch umfangreichere Subvention von Niedrigstlöhnen" durch eine negative Einkommensteuer scharf kritisiert (ver.di 2007). Der DGB hingegen ist offen für eine „direkte oder indirekte Senkung der Sozialabgaben für Arbeitnehmer im Niedriglohnbereich" als Teil eines Konzepts für die Neuordnung des Niedriglohnsektors (Sommer 2007).

\section{Ausblick auf die Tarifrunde 2007}

In einigen Tarifbereichen wurden bereits Tariferhöhungen für das Jahr 2007 vereinbart, wie Übersicht 5 zeigt. Die Steigerungsraten bewegen sich zwischen 1,4 und $3,8 \%$.

Im Jahr 2007 stehen im Übrigen in zahlreichen Wirtschaftszweigen Tarifverhandlungen an. In erster Linie werden die Tarifverhandlungen in der chemischen Industrie und in der Metall- und Elektroindustrie das Tarifgeschehen bestimmen. Die Vergütungstarifverträge in der Chemie 
sind zum Teil bereits Ende 2006 ausgelaufen. In der Metallindustrie reicht die Laufzeit der Verträge bis Ende März 2007. Die IG BCE hat ihre Tarifforderung nicht beziffert, fordert aber deutliche Reallohnsteigerungen unter Berücksichtigung der Produktivitätsentwicklung, die für die Branche mit rund $4,5 \%$ beziffert wird. In der IG Metall zeichnet sich eine Forderung von bis zu $7 \%$ ab.

Ende März folgen das Bauhauptgewerbe (Forderung: 5,5\%), die Druckindustrie, einige regionale Bereiche des Einzel- und Großhandels sowie der Holz und Kunststoff verarbeitenden Industrie. Ende Juni enden die Verträge unter anderem bei der

\begin{tabular}{|lcc|}
\hline $\begin{array}{l}\text { Übersicht 5: Tariferhöhungen in } \mathbf{2 0 0 7} \text { (Erhöhungen aus Abschlüssen } \\
\text { 2006) - in \%- }\end{array}$ \\
\hline Branche & 1,5 & ab \\
\hline Bankgewerbe & 2,5 & $12 / 2007$ \\
Deutsche Post AG & 3,8 & $11 / 2007$ \\
Eisen- und Stahlindustrie & 1,4 & $01 / 2007$ \\
Hotels und Gaststätten NRW & 2,0 & $04 / 2007$ \\
Papierverarbeitung & 1,8 & $08 / 2007$ \\
Privates Verkehrsgewerbe NRW & 2,0 & $05 / 2007$ \\
Textil und Bekleidung & \multicolumn{2}{c|}{ WSI Hans Böckler } \\
\hline & \multicolumn{2}{c}{ Stiftung } \\
\hline
\end{tabular}

Deutschen Bahn AG, Ende Juli bei der Deutschen Telekom AG und Ende August im Versicherungsgewerbe. Im öffentlichen Dienst läuft der Abschluss für Bund und
Gemeinden Ende 2007 aus, der Tarifvertrag für die Länder folgt erst ein Jahr später Ende 2008.

\section{LITERATUR}

BDA Bundesvereinigung der Deutschen Arbeitgeberverbände (2006a): Halbjahresbilanz 2006, Berlin, Juli

BDA Bundesvereinigung der Deutschen Arbeitgeberverbände (2006b): Geschäftsbericht 2006, Berlin, Dezember

BiBB Bundesinstitut für Berufsbildung (2007): Tarifliche Ausbildungsvergütungen 2006 - Weiterhin nur sehr geringer Anstieg, Pressemitteilung 1 vom 4.1.

Bispinck, R. (2006): Mehr als ein Streik um 18 Minuten - Die Tarifauseinandersetzungen im öffentlichen Dienst 2006, in: WSI-Mitteilungen 7, S. 374-381

Bispinck, R./WSI-Tarifarchiv (2006): Tarifpolitischer Halbjahresbericht: Zwischenbilanz der Lohn- und Gehaltsrunde 2006, in: WSI-Mitteilungen 7, S. 359-364

Bofinger, P./Dietz, M./Genders, S./Walwei, U. (2006): Vorrang für das reguläre Arbeitsverhältnis: Ein Konzept für Existenz sichernde Beschäftigung im Niedriglohnbereich, Gutachten für das Sächsische Ministerium für Wirtschaft und Arbeit (SWMA), o. O., August Koalitionsvertrag CDU, CSU und SPD (2005) - Gemeinsam für Deutschland - mit Mut und Menschlichkeit, 11.11., Berlin
Seebacher, K. R. (2006): Der Sozialtarifvertrag, in: Arbeitsrecht im Betrieb, 2

Sommer, M. (2007): Statement auf der DGB-Neujahrspressekonferenz 2007, Berlin, Januar

SPD (2007): Bremer Erklärung der SPD: „In sozialer Verantwortung für unser Land: Für Wachstum, Arbeit und Sicherheit “, 6.1.

Statistisches Bundesamt (2007): Volkswirtschaftliche Gesamtrechnung 2006, vorläufige Ergebnisse, Wiesbaden

SVR Sachverständigenrat zur Begutachtung der gesamtwirtschaftlichen Entwicklung (2006a): Jahresgutachten 2006/07. Widerstreitende Interessen - ungenutzte Chancen, November SVR Sachverständigenrat zur Begutachtung der gesamtwirtschaftlichen Entwicklung (2006b): Arbeitslosengeld II reformieren: Ein zielgerichtetes Kombilohnmodell, Expertise im Auftrag des Bundesministers für Wirtschaft und Technologie ver.di Vereinte Dienstleistungsgewerkschaft (2007): Niedriglohnsubvention. Zum Kombilohnmodell von Professor Bofinger und Dr. Walwei, Wirtschaftspolitische Informationen 1, Berlin, Januar 